\title{
Frozen shoulder: an arthrographic and radionuclear scan assessment
}

\author{
A. I. BINDER, ${ }^{1}$ D. Y. BULGEN ${ }^{1}$ B. L. HAZLEMAN ${ }^{1}{ }^{\text {J }}$. TUDOR, ${ }^{2}$ \\ AND P. WRAIGHT
}

From the Departments of ${ }^{1}$ Rheumatology, ${ }^{2}$ Radiology, and ${ }^{3}$ Nuclear Medicine, Addenbrooke's Hospital, Hills Road, Cambridge CB2 $2 Q Q$

SUMmaRY The diagnostic criteria and nomenclature used to describe the painful stiff shoulder remain confused. Arthrographic features of capsulitis have come to be accepted as characteristic of the frozen shoulder. Increased technetium uptake has also been noted. Both features have been considered to have possible prognostic and therapeutic importance. During a therapeutic study of strictly defined clinical frozen shoulder 35 of 38 patients showed increased technetium diphosphonate uptake in the affected shoulder in comparison with the opposite side. Of 36 patients who had arthrography 15 showed evidence of capsulitis, 11 rupture of the rotator cuff, and five no abnormality. Five tests failed owing to technical difficulty. There was no association between the technetium uptake and the arthrographic features, and neither was useful in predicting the rate or extent of recovery. Frozen shoulder of traumatic onset behaved no differently from that which arose spontaneously. We do not therefore consider that arthrography or technetium diphosphonate scanning performed at presentation contributes to the assessment of the painful stiff shoulder.

The painful stiff shoulder remains a common cause of severe and prolonged disability in middle-aged patients. Frozen shoulder has been used to describe the clinical condition. Neviaser ${ }^{1}$ suggested that adhesive capsulitis was a more suitable name, as his pathological studies revealed chronic inflammation and fibrosis of the joint capsule which became closely adherent to the humeral head. Neviaser ${ }^{2}$ later described the arthrographic features in patients with painful stiff shoulders. Most showed capsulitis with reduced joint volume and obliteration of the inferior and subscapularis recesses. Eleven of his 53 patients, however, showed no arthrographic abnormality. He considered the restriction in range in these patients was due to muscle spasm and that a good response to steroid injections and an early recovery was likely. Reeves ${ }^{3}$ reported arthrography to be useful in differentiating post-traumatic stiff shoulders, with a small but distensible shoulder joint, from frozen shoulder where distensibility was also lost. Treatment and prognosis were considered to be different. Lundberg found a correlation between the severity of range restriction and the

Accepted for publication 19 November 1983.

Correspondence to Dr B. L. Hazleman. reduction in joint volume but recognised that $9 \%$ of patients had a normal arthrogram. Wright and $\mathrm{Haq}^{5}$ found variable features on arthrographaphy of their patients with clinical frozen shoulder and regarded this investigation of little value. However, other authors have considered arthrography of value ${ }^{6-9}$ or even essential ${ }^{10} 11$ in order to make a correct diagnosis and decide on appropriate therapy and likely prognosis.

Minor degenerative changes in both affected and unaffected shoulder joints on $x$-ray are common, ${ }^{12-14}$ and an increased incidence of acromioclavicular arthritis has been reported. ${ }^{5}$ Wright et $a l^{15}$ noted an increased uptake of technetium 99-m pertechnetate in $4 / 10$ patients with frozen shoulder. All four responded to intraarticular steroid, as did one of the negative patients, and it was suggested that this might be a way of identifying steroid responsiveness. Stodell et $a^{16}$ confirmed the increased uptake of pertechnetate but showed a greater uptake if the diphosphonate isotope was used.

Adhesive capsulitis and frozen shoulder are generally regarded as synonymous, and our aim was to assess the plain $x$-ray, arthrographic, and tech- 
netium diphosphonate scan features at presentation in a carefully defined group of patients with clinical frozen shoulder and examine the relevance to the rate and extent of recovery. We also wanted to ascertain if a traumatic onset affected these findings.

\section{Patients and methods}

The 42 patients described in the prospective clinical study were included in this study. Criteria for diagnosis and methods of assessment have already been described. ${ }^{17}$ Forty of the 42 patients were reviewed between 40 and 48 months (mean 44 months) after presentation.

Plain $x$-rays of both shoulders were taken in all 42 patients. Thirty-eight of the patients also had 99-m technetium methylene diphosphonate scans performed before any treatment was given and usually on the same day and immediately before the arthrogram. Three hours after the injection of 10-15 $\mathrm{mCi}$ of 99-m technetium diphosphonate an Elscint wholebody scanner was used to search for the hottest spot over each shoulder. The count was read with a 5 -inch $(12.7 \mathrm{~cm})$, crystal and the ratio of the counts at the affected/unaffected shoulder (the uptake ratio) was calculated. Forty patients of similar age and sex with no shoulder complaints but having diphosphonate scans for unrelated reasons also had counts taken over both shoulders to provide a control group. The count showed little variation between shoulders whether dominant or non-dominant, so control ratios were obtained from uptake in the right/left shoulders.

Thirty-six of the patients had double contrast arthrography performed via the anterior route. A maximum of $5 \mathrm{ml}$ of Conray 280 and $5-15 \mathrm{ml}$ of filtered air were introduced into the shoulder joint under fluoroscopic control. The procedure was always performed within one week of presentation and also before treatment had begun.

\section{Results}

Plain $x$-rays of the affected shoulders (Table 1) were normal in 30 of the 42 patients $(71 \%)$. The remainder showed various minor changes, including a decrease in the distance between the acromion and the humeral head (five patients) and minor degenerative changes in the humeral head which involved the greater tuberosity but not the glenohumeral joint margin (eight patients). Only four patients had radiological abnormalities of the unaffected shoulder. Eight of the patients were manual workers and in 11 the disease had a traumatic onset, but neither factor was associated with an increased incidence of degenerative changes.
Table 1 Shoulder $\mathrm{x}$-ray features (42 patients)

\begin{tabular}{lll}
\hline & $\begin{array}{l}\text { Affected } \\
\text { shoulder }\end{array}$ & $\begin{array}{l}\text { Unaffected } \\
\text { shoulder }\end{array}$ \\
\hline Normal & 30 & 37 \\
$\begin{array}{l}\text { Minor degenerative changes in } \\
\text { humeral head }\end{array}$ & 8 & 2 \\
$\begin{array}{l}\text { Supraspinatus tendon calcification } \\
\text { Degeneration of acromioclavicular } \\
\text { joint }\end{array}$ & 2 & 0 \\
$\begin{array}{l}\text { Narrowing of acromion/humeral } \\
\text { head distance }\end{array}$ & 3 & 1 \\
\hline
\end{tabular}

\section{ARTHROGRA PHY}

Although this investigation was attempted in 36 patients, four failed owing to technical difficulty, and one patient withdrew from the study as a result of severe pain which followed the arthrography. Five other patients reported the injection to be painful, and most noted some exacerbation of shoulder pain, which began after 4-6 hours and lasted 24-72 hours.

Of the 31 patients who had successful arthrography three different subgroups emerged.

(1) Normal group (five patients). The arthrogram was accepted as normal if it revealed a distensible shoulder joint with a smooth regular outline and a volume of at least $15 \mathrm{ml}$. An inferior and subscapularis recess was usually noted and contrast medium entered the bicipital tendon sheath but not the subacromial bursa.

(2) Capsulitis group (15 patients). A marked reduction in joint volume (often under $5 \mathrm{ml}$ ) with loss of distensibility of the shoulder joint was found. Marked irregularity of joint outline (Fig. 1) and early lymphatic filling was also sometimes seen.

(3) Rupture group (11 patients). Rupture of the rotator cuff was considered to be present in an immediate flow of contrast medium from the shoulder joint into the subacromial/subdeltoid bursa (Fig. 2) occurred. With contrast medium present in the shoulder joint and subacromial bursa, the rotator cuff was clearly visible above the humeral head. In three patients the rupture was small and revealed a normal looking joint. In the other eight patients it was not possible to outline the joint sufficiently to assess detail. All five patients with a decrease in the distance between the acromion and the humeral head showed rupture of the rotator cuff.

\section{TECHNETIUM SCANS}

Thirty-five of the 38 patients had an increased technetium diphosphonate uptake (Fig. 3) in the affected shoulder - i.e., uptake ratio greater than 1 - in comparison to the opposite side, 11 of these 


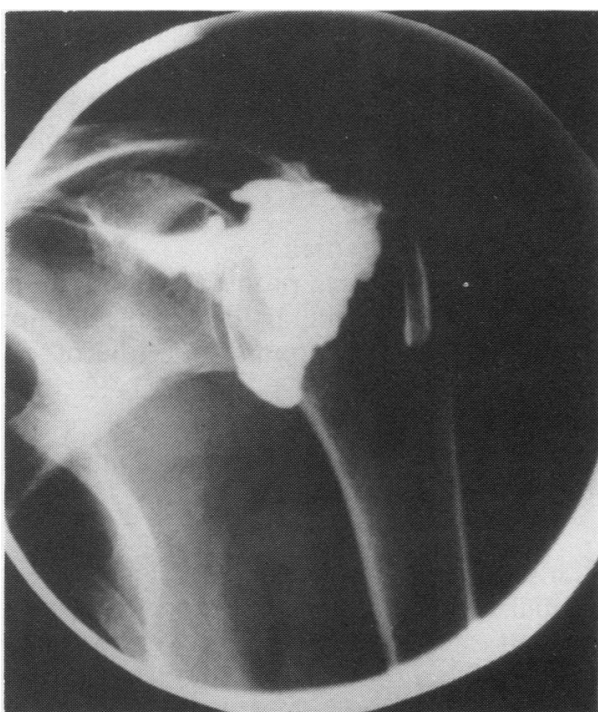

Fig. 1 Arthrogram showing marked irregularity of joint outline and early lymphatic filling.

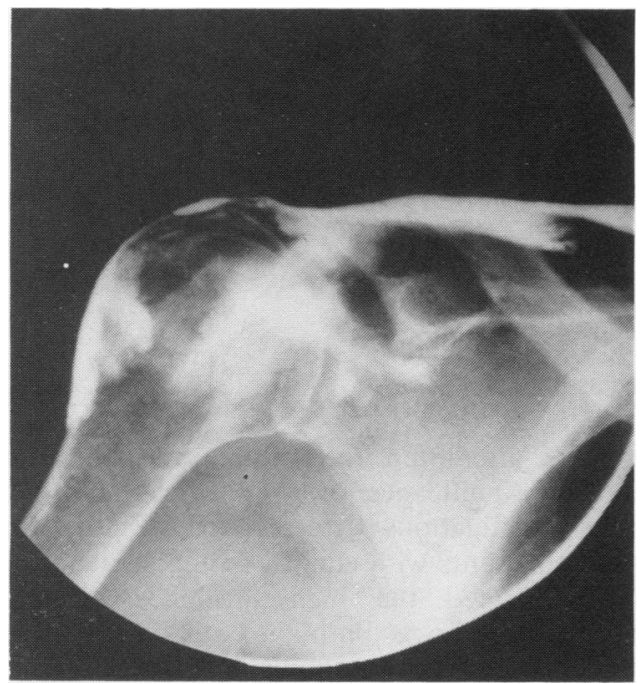

Fig. 2 Arthrogram of rupture of the rotator cuff showing contrast medium present in shoulder joint and subacromial bursa. The rotator cuff is visible above the humeral head.

scans showing more than a $50 \%$ increase in uptake. Comparison of the uptake in the two shoulders (Student's $t$-test) showed the increase in uptake by the affected shoulder to be significant $(p \leqslant 0 \cdot 0001)$. The distribution of the uptake ratios (Fig. 4) in the patients showed a mean of $1 \cdot 31$ and in the controls $1 \cdot 04$, the difference being significant $(p \leqslant 0 \cdot 001$, Student's $t$-test). Duration of symptoms at presentation did not influence the result. No correlation was 


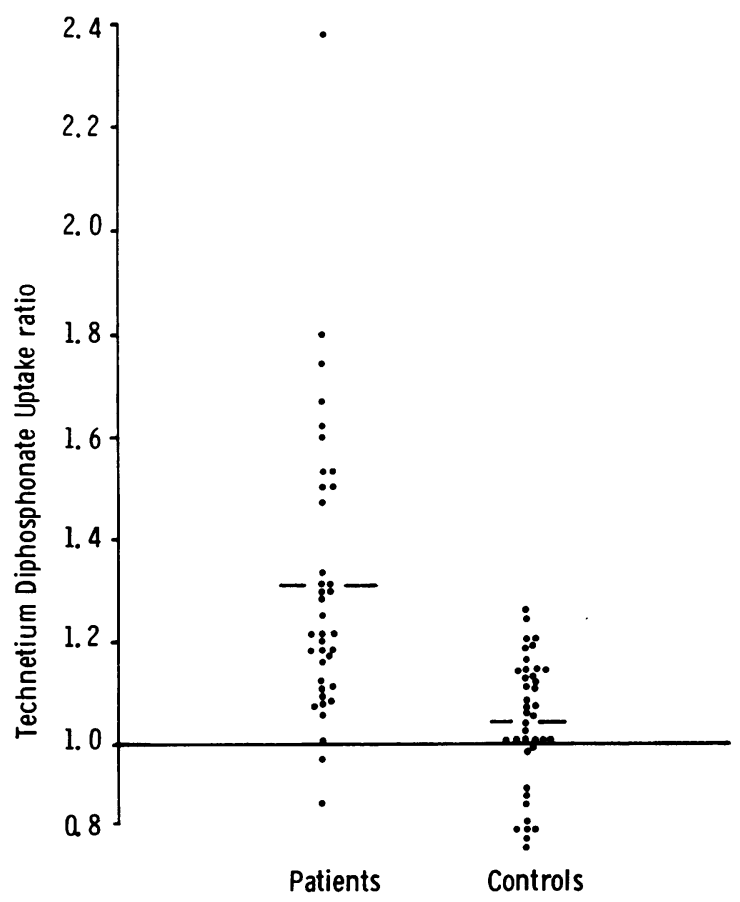

Fig. 4 Technetium uptake ratios in frozen shoulder (affected/unaffected) and controls (right $v$. left).

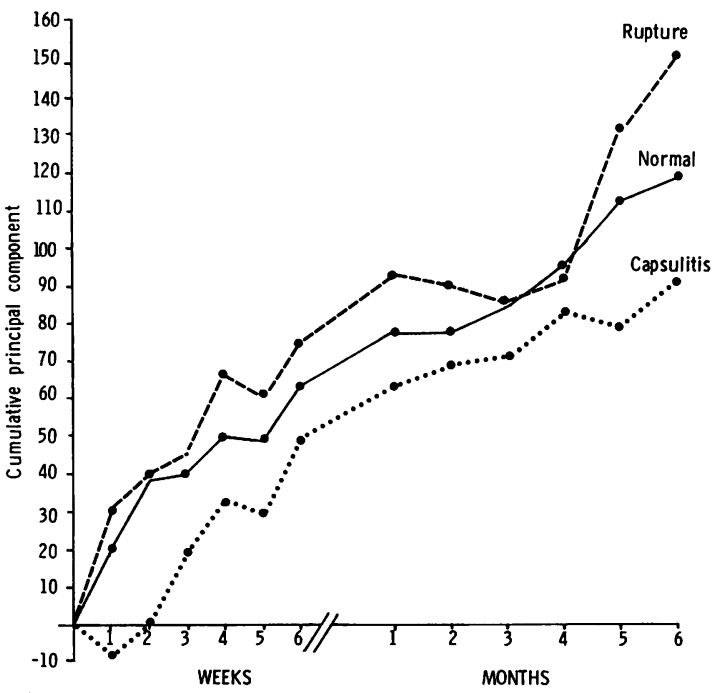

Fig. 5 The relationship between the arthrogram finding and the cumulative principal component.

suggests that the capsulitis group recovered more slowly in the initial weeks. By three months the range was similar in each group. Analysis of variance showed no significant difference between the arthrographic groups at presentation and no significant difference in range or rate of recovery during follow-up. No association was found between the passive range or its recovery and the findings on plain $x$-ray or technetium scan.

\section{DURATION OF SYMPTOMS}

The mean duration of symptoms at presentation was significantly longer in the arthrographically normal group (9.0 range 3-12 months) than the other two arthrographic groups (3.76 range 1-12 months) $(p \leqslant 0.0092$, analysis of variance). As previously mentioned, duration of symptoms did not influence the technetium uptake.

TRA UMATIC ONSET

The initial severity of pain and range limitation and subsequent rate and extent of recovery were similar in patients with a spontaneous and traumatic onset. No features were noted at arthrography or on scanning which suggested the mode of onset.

\section{LATE REVIEW (mean 44 months)}

While half the patients in the capsulitis and rupture groups still had mild discomfort or restriction of movement at review, all five patients from the 'normal group' had some disability, though this was only severe in one patient. The range of individual movements also showed a less satisfactory outcome in the 'normal group', but the difference was not significant.

\section{Discussion}

We have attempted to evaluate the initial and long-term significance of radiological and radioisotope investigations performed in a strictly defined group of patients with clinical frozen shoulder. We have confirmed the high incidence of minor degenerative changes on plain $x$-ray of the affected shoulder ${ }^{5-15}$ but not shown a high incidence of change in the acromioclavicular joint ${ }^{5}$ or opposite shoulder. The only $x$-ray finding of practical value was the reduction in distance between the acromion and humeral head, which reliably predicted rupture of the rotator cuff.

We have confirmed ${ }^{16}$ the increased uptake of technetium diphosphonate by the painful stiff shoulder in comparison with the opposite side or controls, but we have. been unable to show any correlation with the duration of symptoms, initial severity, or recovery. Patients with a marked increase in technetium uptake showed no better response to therapy than those with little or no increase. The explanation for the increased uptake 
of the diphosphonate ('bone-seeking') isotope remains unclear. Only two of our patients had macroscopic calcification of the supraspinatus tendon on plain $x$-ray. Microscopic calcium hydroxyapatite crystal deposition, increased vascularity, ${ }^{18}$ and interaction between collagenous structures and microcrystalline complexes containing calcium $^{15}$ have been suggested.

Our shoulder arthrography results differ markedly from those in many other reported series where the features of capsulitis are said to be always present. Kernwein et al, ${ }^{19}$ Samilson et al, ${ }^{20}$ and Preston and Jackson ${ }^{8}$ found capsulitis in all their patients but provided little clinical detail. Neviaser ${ }^{11}$ regarded arthrography as essential for the diagnosis of adhesive capsulitis, but the clinical studies did not show that the patients with normal arthrography in his initial study ${ }^{2}$ had a better prognosis than the clinically identical patients showing capsulitis. Reeves $^{21}$ showed that arthrograms performed in the convalescent phase after the recovery of range had returned to normal. Our normal group did have a significantly longer mean duration at presentation, but their range was just as restricted as the other arthrographic groups. The rate and extent of recovery was also no different in patients with a normal arthrogram; consequently we do not feel that we can attribute the lack of arthrographic abnormality in these patients solely to the duration of symptoms at presentation. It is unclear if our patients with a traumatic onset are similar to those described by Reeves, ${ }^{3}$ as he has given little clinical detail. However, we have not been able to show any difference in prognosis between a traumatic and a spontaneous onset. We have also been unable to show any association between the onset (traumatic or spontaneous) and the features at arthrography. As only half our patients showed evidence of capsulitis at arthrography, we did not attempt to confirm the reported correlation ${ }^{4}$ between range restriction and joint volume.

The variability in the features at arthrography and technetium uptake may reflect differences in the underlying pathology, but were not useful in predicting outcome. We do not therefore consider that either procedure is of value in the routine assessment of the painful stiff shoulder.

\section{References}

1 Neviaser J S. Adhesive capsulitis of the shoulder: a study of the pathological findings in periarthritis of the shoulder. $J$ Bone Joint Surg 1945: 27A: 211-21.

2 Neviaser J S. Arthrography of the shoulder joints: study of the findings in adhesive capsulitis of the shoulder. J Bone Joint Surg 1962: 44A: $1321-30$.

3 Reeves B. Arthrographic changes in frozen and post-traumatic stiff shoulders. Proc $R$ Soc Med 1966; 59: 827-30.

4 Lundberg BJ. The frozen shoulder. Acta Orthop Scand 1969; suppl 119.

5 Wright V. Haq AMMM. Periarthritis of the shoulder. Ann Rheum Dis 1976; 35: 220-6.

6 Weiss J J. Thompson G R. Doust V. Burgener F A. Arthrography in the diagnosis of shoulder pain and immobility. Arch Phys Med Rehabil 1974; 55: 205-9.

7 Nelson C H. Burton R I. Upper extremity arthrography. Clin Orthop 1975; 107: 62-72.

8 Preston B J. Jackson J P. Shoulder arthrography. Br J Radiol 1976; 49: 266.

9 Rizk T E. Pinals R S. Frozen shoulder. Semin Arthritis Rheum 1982: 11: 440-52.

10 Weiss $\mathrm{J}$ J. Ting Y M. Arthrography-assisted intra-articular injection of steroid in the treatment of adhesive capsulitis. Arch Phys Med Rehabil 1979; 59: 285-7.

11 Neviaser J S. Adhesive capsulitis and the stiff and painful shoulder. Orthop Clin N Am 1980; 11: 327-31.

12 Olsson O. Degenerative changes of the shoulder joint and their connection with shoulder pain. Acta Chir Scand 1935; suppl 181 .

13 Golding F C. The shoulder-the forgotten joint. $\mathrm{Br} J$ Radiol 1962; 35: 149-58.

14 Cotton R E. Rideout D F. Tears of the humeral rotator cuff. A radiological and pathological necropsy survey. $J$ Bone Joint Surg 1964: 46B: 314-28.

15 Wright M G. Richards A J. Clarke M B. 99-m pertechnetate scanning in capsulitis. Lancet 1975; ii: 1265.

16 Stodell M A. Nicholson R. Scott J, Sturrock R D. Radioisotope scanning in the painful shoulder. Rheumatol Rehabil 1980; 19: 163-6.

17 Bulgen D Y, Binder A I, Hazleman B L. Dutton J, Roberts S. Frozen shoulder: prospective clinical study with an evaluation of three treatment regimens. Ann Rheum Dis (same issue).

18 Guillemart A. Besnard J C. LePape A, Gally G, Fetiffoff F. Skeletal uptake of pyrophosphate labelled with technetium-95m and technetium-96 as evaluated by autoradiography. $J \mathrm{Nucl}$ Med Allied Sci 1978; 19: 895-9.

19 Kernwein G A. Roseberg B. Sneed W R. Arthrographic studies of the shoulder joint. J Bone Joint Surg 1957: 39A: 1267-79.

20) Samilson R L. Raphacl R L. Post L. Noonan C. Siris E. Rancy Jr F L. Shoulder arthrography. JAMA 1961: 175: 773-9.

21 Reeves B. The natural history of the frozen shoulder syndrome. Scand J Rheumatol 1975: 4: 193-6. 